\title{
A New Method for a Mutagenicity Test Using Spectrophotometry
}

\author{
Jinzhong Zhang, Wanzhi WeI, ${ }^{\dagger}$ Youan Mao, Wei Lan, and Mingjun Chen \\ College of Chemistry and Chemical Engineering, Hunan University, Changsha, 410082, P. R. China
}

\begin{abstract}
A new method for a mutagenicity test with spectrophotometry is presented in this paper. The mutagenic activity was examined by monitoring the growth situation of the Salmonella typhimurium TA98 strain. It was found that this strain could mutate and regain its growth capability owing to the impact of dimethyl sulfate; thus, metabolites of the strain caused the culture system to exhibit turbidness. The optimum experimental conditions were obtained. The effect of the dose of the mutagen on the absorbance was also studied. The result showed that there was a good linear relationship between the absorbance and the dose of the mutagen over the range of $2.5-20 \mu \mathrm{g} / \mathrm{ml}$; the regression equation was $A=$ $1.27 \times 10^{-3}+0.0134 C$, where $A$ is the absorbance and $C$ in $\mu \mathrm{g} / \mathrm{ml}$ is the concentration of the mutagen. This method requires no immobilization or preincubation of the microorganism, and can be directly applied to mutagen detection. It is thus rapid and convenient.
\end{abstract}

(Received May 1, 2000; Accepted October 4, 2000)

\section{Introduction}

Ames et $a l .{ }^{1}$ found that about 90 percent of carcinogens have mutagenicity after they had studied some known chemical carcinogens. This result proved that there is a correlation between the carcinogenic activity and the mutagenicity. Thus, people can predict the carcinogenic activity of chemical pollutants by examining their mutagenic activity. Thus, a mutagenicity test has an important meaning in environmental medicine. A large number of research results have shown that the chemical components in airborne particulate matter, ${ }^{2,3}$ contaminated soil ${ }^{4,5}$ and drinking water ${ }^{6,7}$ can be mutagenic. In order to protect public health, many researchers have tried to develop mutagenicity test methods. Some methods using microorganisms have been proposed, such as the Ames Salmonella/mammalian microsome test (Ames test), ${ }^{8,9}$ the microbial bioluminescence method (mutatox test), ${ }^{10}$ microdose forward mutagenic method ${ }^{11}$ and the microbial sensor methods. ${ }^{12,13}$ However, the Ames test requires a two-day culture time to obtain test results, microbial sensor methods ${ }^{12,13}$ require the immobilization and preincubation of microorganisms, and the operations of other methods are troublesome. Therefore, it is essential to develop a rapid and convenient test method for mutagens.

In this study, the mutagenic activity was examined by monitoring the bacterial growth situation with spectrophotometry. The method was based on the fact that the Salmonella typhimurium auxotroph strain (TA98) could not grow without histidine, and that no absorption could be detected. If a mutagen was added into the culture medium of the strain, the strain could happen to mutate and revert to its ancestral type, ${ }^{8}$ and the culture system became turbid due to growth of the strain. Thus, optical absorption could be caused

† To whom correspondence should be addressed.

J. Z.: on leave from Southwest Agricultural University, Chongqing, China.

E-mail: zhangjinzhong@cmmail.com. at a certain wavelength, and a mutagenicity test could be performed with spectrophotometry. Dimethyl sulfate was used for a mutagenic study in this work, since it is a typical alkyl carcinogen. ${ }^{14}$

Compared with other reported methods, ${ }^{8-13}$ this method requires no immobilization or preincubation of microorganism, and can be used to detect mutagen directly. Therefore, the proposed method is both rapid and convenient.

\section{Experimental}

\section{Instruments}

A thermostat incubator (Model PYX-DHS-50 $\times 60$, Yuejin medical instrument factory of Shanghai, China) was used to incubate the bacterial solution. A spectrometer (Model 721, the third analytical instrument factory of Shanghai, China) was used to determine the absorbance of the culture system.

\section{Reagents and solutions}

Dimethyl sulfate (DMS) was of analytical grade, and purchased from the first analytical reagent factory of Tianjin (Tianjin, China). DMS solution $(0.5 \mathrm{mg} / \mathrm{ml})$ was prepared by dissolving $0.25 \mathrm{ml}$ of dimethyl sulfate (DMS) in $1 \mathrm{ml}$ of ethanol, which was then diluted to $100 \mathrm{ml}$ with distilled water; a $15 \mathrm{ml}$ volume of this solution was diluted to $100 \mathrm{ml}$ with distilled water.

Histidine as a biochemical reagent was of chromatographic grade, and purchased from Dongfeng biochemical technological corporation, Shanghai. A histidine solution $(1 \mathrm{mg} / \mathrm{ml})$ was prepared by dissolving $10 \mathrm{mg}$ of histidine in $10 \mathrm{ml}$ of distilled water, which was then sterilized by autoclaving at $121^{\circ} \mathrm{C}$ for 15 $\min$.

A phosphate buffer solution (PBS) of $\mathrm{pH} 7.0$ was prepared with $50 \mathrm{ml}$ of a $0.1 \mathrm{~mol} / 1 \mathrm{KH}_{2} \mathrm{PO}_{4}$ solution and $29.1 \mathrm{ml}$ of a 0.1 $\mathrm{mol} / \mathrm{l} \mathrm{NaOH}$ solution, then diluted to $100 \mathrm{ml}$ with distilled water. The preparation of PBS ( $\mathrm{pH} 7.2)$ was the same as that of PBS ( $\mathrm{pH} 7.0$ ), except that the volume of $0.1 \mathrm{~mol} / \mathrm{l} \mathrm{NaOH}$ solution was $34.7 \mathrm{ml}$. 
All other reagents used were of analytical grade. Doubly distilled and sterilized water was used throughout.

\section{Culture media}

The composition of the medium ${ }^{15}$ for Salmonella typhimurium TA98 was as follows: glucose, $20.0 \mathrm{~g}$; citric acid, $2.0 \mathrm{~g}$; $\mathrm{K}_{2} \mathrm{HPO}_{4} \cdot 3 \mathrm{H}_{2} \mathrm{O}, 3.5 \mathrm{~g} ; \mathrm{MgSO}_{4} \cdot 7 \mathrm{H}_{2} \mathrm{O}, 0.2 \mathrm{~g}$; and distilled water, $1000 \mathrm{ml}$. The composition of the broth medium was as follows: beef extract, $5 \mathrm{~g}$ : peptone, $10 \mathrm{~g}$; $\mathrm{NaCl}, 5 \mathrm{~g}$; and distilled water, $1000 \mathrm{ml}$. Both media were sterilized by autoclaving at $121^{\circ} \mathrm{C}$ for $15 \mathrm{~min}$.

\section{Bacterial solution}

The Salmonella typhimurium TA98 strain (incubated on broth-agar slant) was obtained commercially (Beijing, China). Four loops of TA98 on an agar slant were inoculated into 100 $\mathrm{ml}$ of a broth medium ( $\mathrm{pH} 7.2)$ using an inoculating loop ( $2 \mathrm{~mm}$ in diameter), and then incubated at $37^{\circ} \mathrm{C}$ for $16 \mathrm{~h}$ under a condition providing oxygen. The mixture was then preserved in a refrigerator at $4^{\circ} \mathrm{C}$. The culture gave an approximate concentration of $3.7 \times 10^{3}$ cells $/ \mathrm{ml}$, detected by the pour plate count (PPC) method. The concentration represented the number of live bacteria, and the major bacteria remained in a latent state of growth.

\section{Procedures}

Detection of the absorption spectrum curve of the culture solution. The culture solution was prepared by mixing with 5 $\mathrm{ml}$ of the culture medium, $0.5 \mathrm{ml}$ of histidine solution, $5 \mathrm{ml}$ of TA98 bacterial solution $\left(18.5 \times 10^{3}\right.$ cells $), 10 \mathrm{ml}$ of PBS $(\mathrm{pH}$ 7.0) and oxygen-saturated distilled water to $50 \mathrm{ml}$ as a final volume. Also, the culture solution was incubated at $37^{\circ} \mathrm{C}$ for 5 $\mathrm{h}$ in an incubator. The absorbance was then detected in a $2-\mathrm{cm}$ cuvette over the range $360-560 \mathrm{~nm}$ by spectrophotometry. The number of parallel determinations was 3 , and the mean value of absorbance $(A) v s$. wavelength $(\lambda)$ was plotted. The maximum absorption wavelength $\left(\lambda_{\max }\right)$ was obtained through the $A-\lambda$ curve.

Monitoring of the normal growth of bacteria. The preparation of a culture solution was the same as that in detecting the $A-\lambda$ curve; the absorbance was detected at $\lambda_{\max }$ with a certain time interval by spectrophotometry. The number of parallel determinations was 3 .

Monitoring of the mutagenic case. The preparation of a culture solution was the same as that in detecting the $A-\lambda$ curve, except that a 1-ml DMS solution was added instead of histidine; the absorbance was detected at $\lambda_{\max }$ with a certain time interval by spectrophotometry. The number of parallel determinations was 3.

The effect of different experimental conditions on the absorbance was examined, such as the culture temperature, the initial concentration of the bacterial solution, the $\mathrm{pH}$ value (adjusted by using a $0.5 \mathrm{~mol} / \mathrm{l}$ of $\mathrm{HCl}$ solution or $0.5 \mathrm{~mol} / \mathrm{l}$ of a $\mathrm{NaOH}$ solution) and the volume of the culture medium. Thus, the optimum experimental conditions were obtained.

Under the selected experimental conditions, the absorbance values were detected in the presence of different doses of the mutagen.

\section{Results and Discussion}

The absorption spectrum curve of the culture solution

The absorption spectrum curve of the culture solution in the presence of histidine is shown in Fig. 1. It can be found that the

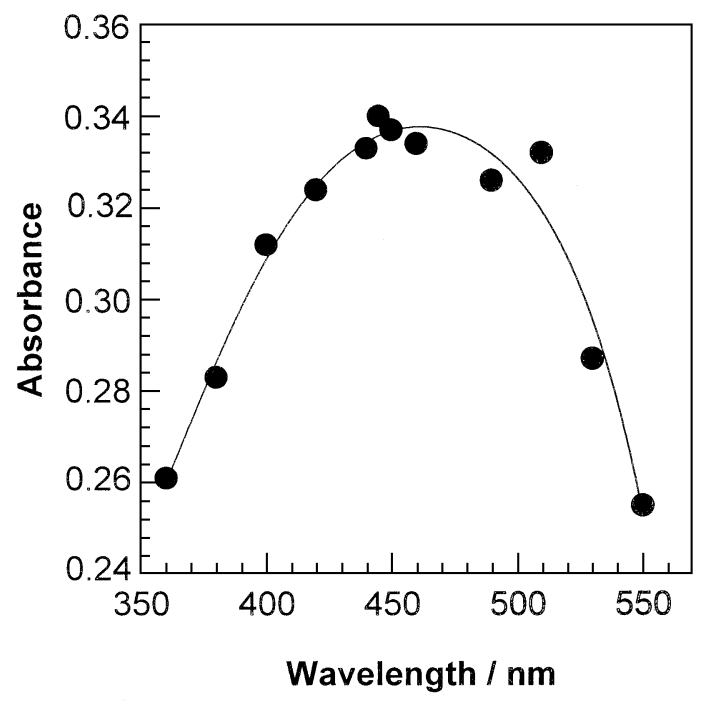

Fig. 1 Absorption spectrum curve of the culture solution.

absorbance increases with an increase in the wavelength over the range $360-445 \mathrm{~nm}$, decreases in the range $445-560 \mathrm{~nm}$, and reaches the maximum value $(0.34)$ at $445 \mathrm{~nm}$. This result illustrates that the culture solution has the maximum extent of absorption at $445 \mathrm{~nm}$. Thus, $445 \mathrm{~nm}$ was chosen as the detection wavelength throughout the experiment.

\section{Monitoring of the normal growth of bacteria}

A curve of absorbance $(A) v s$. culture time $(t)$ of the normal growth of the bacteria is shown by curve 1 in Fig. 2. Because experiments showed that the auxotroph strain could not grow in the absence of histidine, and no absorption could be detected, this culture solution was used as a reference solution. When histidine was added into the culture medium, the bacteria in the latent state began to grow, and displayed normal growth, which led to a change in the absorbance. From curve 1, it can be illustrated that no absorbance could be detected in the first 0.5 $\mathrm{h}$, and that the absorbance then increases from 0.5 to $3 \mathrm{~h}$, finally reaching a stable level. It should be noted that the slope of the $A-t$ curve resembles the theoretical growth curve of bacteria. The section of the $A-t$ curve in the initial $0.5 \mathrm{~h}$ corresponds to the lag period of the strain, the section from 0.5 to $3 \mathrm{~h}$ corresponds to the exponential growth period, and after 3 h's incubation, the bacterial growth reaches saturation. From curve 1 , an increased value of $c a$. 0.34 in the absorbance could be obtained during the exponential growth period.

\section{Monitoring of the mutagenic case}

The $A-t$ curve of the mutagenic case is shown by curve 2 in Fig. 2. It can be seen that no absorption can be detected in the first $1.5 \mathrm{~h}$; the absorbance then increases from 1.5 to $5 \mathrm{~h}$, finally reaching a constant value, basically. As shown in curve 2 , an increased value of $c a$. 0.16 in absorbance could be obtained during the exponential growth period.

Compared with curve 1 , the result in curve 2 displays a similar changing trend of the absorbance. It can be demonstrated that the strain happens to mutate and revert to the ancestral type owing to the impact of DMS, and thus shows a similar growth situation in the presence of histidine. Moreover, one could investigate the absorbance in the two curves from three aspects. First, because the time that the absorbance begins to increase is different, the lag time expressed by the bacteria is different. It testifies that the auxotroph strain needs a period of 


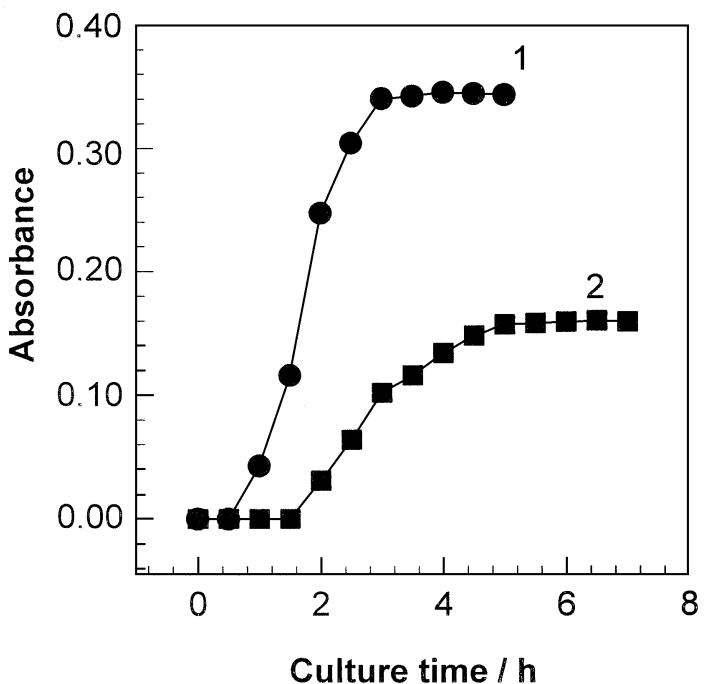

Fig. 2 Absorbance-time curves in different cases. 1, Normal growth curve of the strain; 2, mutagenic curve of the strain.

Table 1 Bacterial concentrations of normal growth and mutagenic case at different culture time

\begin{tabular}{ccc}
\hline Culture time/h & $\begin{array}{c}\text { Bacterial concentration } \\
\text { of normal growth } \\
\text { (cells/ml) }\end{array}$ & $\begin{array}{c}\text { Bacterial concentration } \\
\text { of mutagenic case } \\
\text { (cells/ml) }\end{array}$ \\
\hline 0 & $3.7 \times 10^{2}$ & $3.7 \times 10^{2}$ \\
0.5 & $3.7 \times 10^{2}$ & $3.7 \times 10^{2}$ \\
1.0 & $4.0 \times 10^{4}$ & $3.7 \times 10^{2}$ \\
1.5 & $1.1 \times 10^{5}$ & $3.7 \times 10^{2}$ \\
2.0 & $2.3 \times 10^{5}$ & $2.9 \times 10^{4}$ \\
2.5 & $2.8 \times 10^{5}$ & $6.0 \times 10^{4}$ \\
3.0 & $3.2 \times 10^{5}$ & $9.5 \times 10^{4}$ \\
3.5 & $3.2 \times 10^{5}$ & $1.1 \times 10^{5}$ \\
4.0 & $3.2 \times 10^{5}$ & $1.2 \times 10^{5}$ \\
4.5 & & $1.4 \times 10^{5}$ \\
5.0 & & $1.5 \times 10^{5}$ \\
5.5 & & $1.5 \times 10^{5}$ \\
6.0 & & $1.5 \times 10^{5}$ \\
\hline
\end{tabular}

time to regain its growth capability. In this way, one can distinctly observe a prolongation of the lag time, and bacterial growth caused by mutation is slower than normal growth. Thus, the lag time could be used as a criterion of the intensity of mutation. Secondly, the increased value of the absorbance is different during the exponential growth period. This may indicate that the number of induced revertants produced by the mutation is related to the mutagenic activity of chemical substance, ${ }^{8,16}$ and less than that of the normal-growth bacteria. Therefore, the amount of metabolites in the mutagenic case is less and the turbidity is lower than that of normal growth Thirdly, the slope of the $A-t$ curve is different during the exponential-growth period. It should be found that the slope of the $A-t$ curve in the mutagenic case is smaller and the duration of exponential growth is longer than that of normal growth. These phenomena also testify that the bacterial growth caused by mutation is slower than the normal growth. In this study, $5 \mathrm{~h}$ was chosen as the culture time in the mutagenic case.

To examine the bacterial growth situation, we detected bacterial concentrations during the course of the photometry at different culture times for normal growth and mutagenic cases;

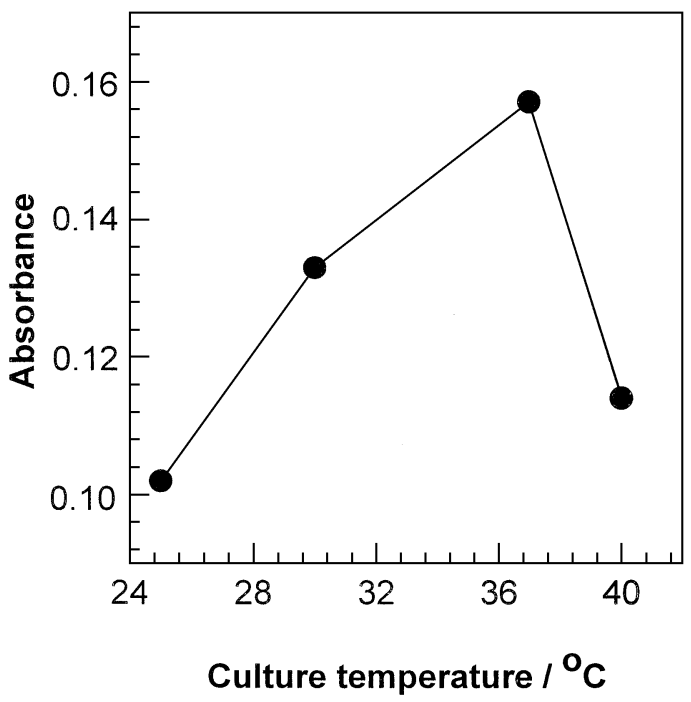

Fig. 3 Effect curve of the culture temperature on the absorbance. The other experimental conditions are the same as curve 2 in Fig. 2, except for the culture temperature.

the results are given in Table 1. It could be found that in the exponential-growth phase, the bacterial concentration increases along with an increase in the culture time. Also, the bacterial concentration in the normal-growth case is more than that in the mutagenic case for the same culture time. These results demonstrate that it is feasible to study mutagenicity with spectrophotometry.

\section{Effect of the culture temperature}

The effect curve of different culture temperatures on absorbance is shown in Fig. 3. It can be found that the absorbance increases along with an increase in the temperature, reaches the maximum value at $37^{\circ} \mathrm{C}$ and decreases at $40^{\circ} \mathrm{C}$. This result is consistent with the growth rule of bacterium, because it is beneficial for the strain to mutate and acquire more revertants at high temperature. However, because it is unfavorable to the growth of a strain when the temperature is $40^{\circ} \mathrm{C}$, the absorbance decreases. To shorten the culture time, and approach the condition of mutagen impacting on the human body, $37^{\circ} \mathrm{C}$ was selected as the culture temperature.

\section{Effect of the initial concentration of a bacterial solution}

The effect curve of different initial concentrations of a bacterial solution on the absorbance is shown in Fig. 4. It can be seen that the absorbance of the culture solution increases along with an increase in the initial concentration of the bacterial solution. This may indicate that the more induced revertants exist in a greater amount of bacterial solution, and that their rapid growth causes the absorbance to greatly increase. It should be noted from Fig. 4 that a greater absorbance value can be obtained when the initial bacterial concentration is $3.7 \times 10^{2}$ cells $/ \mathrm{ml}$. This result shows that distinct mutation occurs in the culture system. As a result, an initial concentration of the bacterial solution in the culture system of $3.7 \times 10^{2}$ cells $/ \mathrm{ml}$ was used in the mutagenicity test.

\section{Effect of the $\mathrm{pH}$ value on the absorbance}

The effect curve of different $\mathrm{pH}$ values on the absorbance is shown in Fig. 5. It can be found that the absorbance increases when the $\mathrm{pH}$ value is from 5 to 7 , but decreases when it is from 7 to 9 , and reaches the maximum value when the $\mathrm{pH}$ is 7 . This 


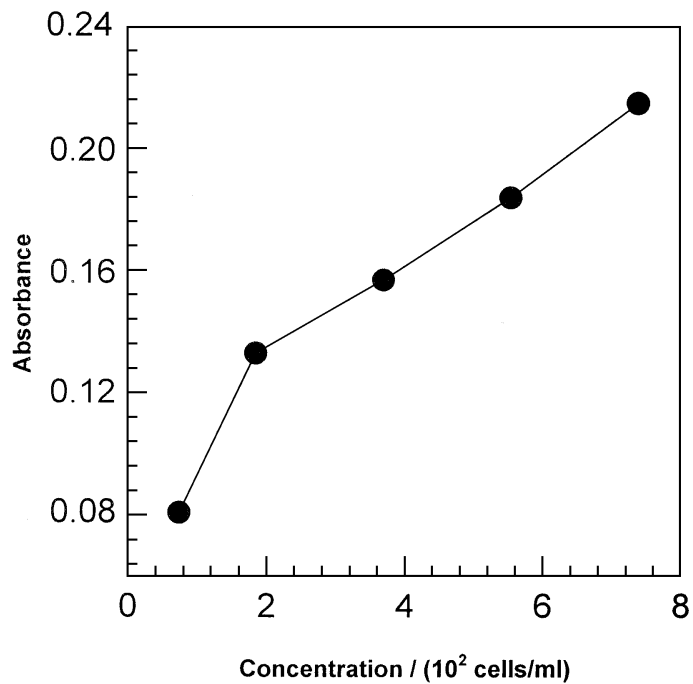

Fig. 4 Effect curve of the initial concentration of a bacterial solution on the absorbance. The other experimental conditions are the same as curve 2 in Fig. 2, except for the initial concentration of the bacterial solution.

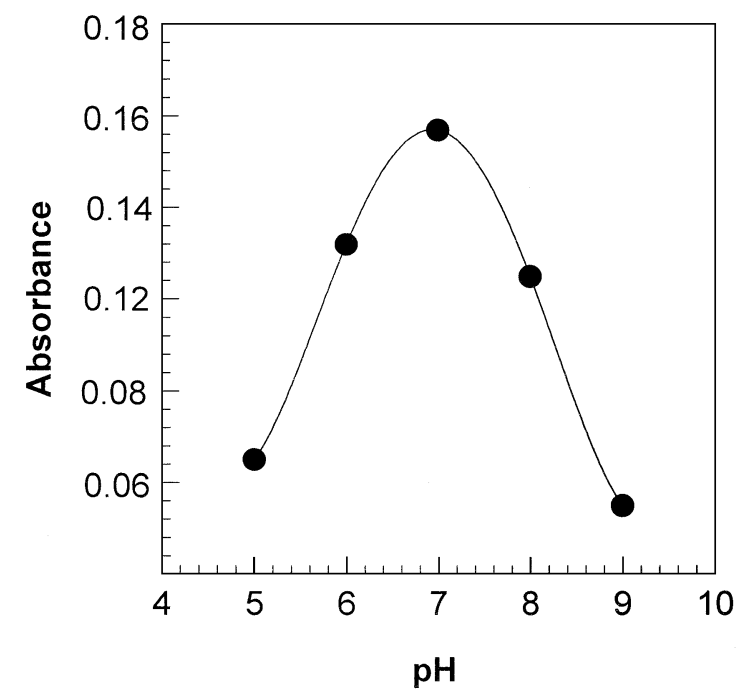

Fig. 5 Effect curve of the $\mathrm{pH}$ value on the absorbance. The other experimental conditions are the same as curve 2 in Fig. 2, except for the $\mathrm{pH}$ value.

result demonstrates that a good mutagenic effect is obtained when the $\mathrm{pH}$ value is 7 . This may indicate that the strain is suitable to grow in a neutral environment. ${ }^{13}$ Also, a $\mathrm{pH}$ of 7 was chosen as the medium condition in the mutagenicity study.

\section{Effect of the amount of culture medium}

The effect curve of different amounts of culture medium on the absorbance is shown in Fig. 6. It can be seen that in a certain range of culture medium, the absorbance increases along with an increase in the amount of culture medium. This result demonstrates that more nutrient substances are beneficial to the growth of revertants. Because a good growth effect of the strain is obtained when the volume of culture medium is $5 \mathrm{ml}, 5 \mathrm{ml}$ of the culture medium was used in the mutagenicity test.

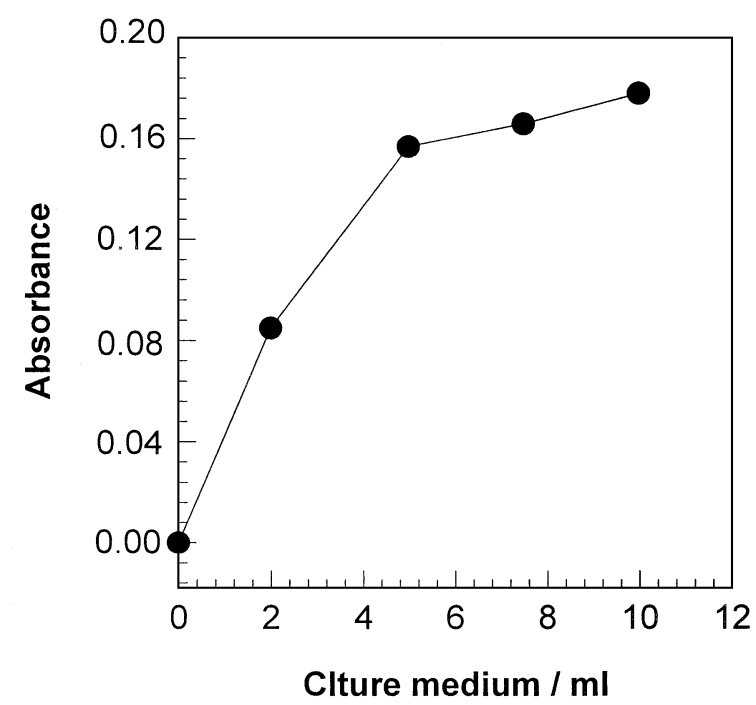

Fig. 6 Effect curve of the amount of the culture medium on the absorbance. The other experimental conditions are the same as curve 2 in Fig. 2, except for the amount of culture medium.

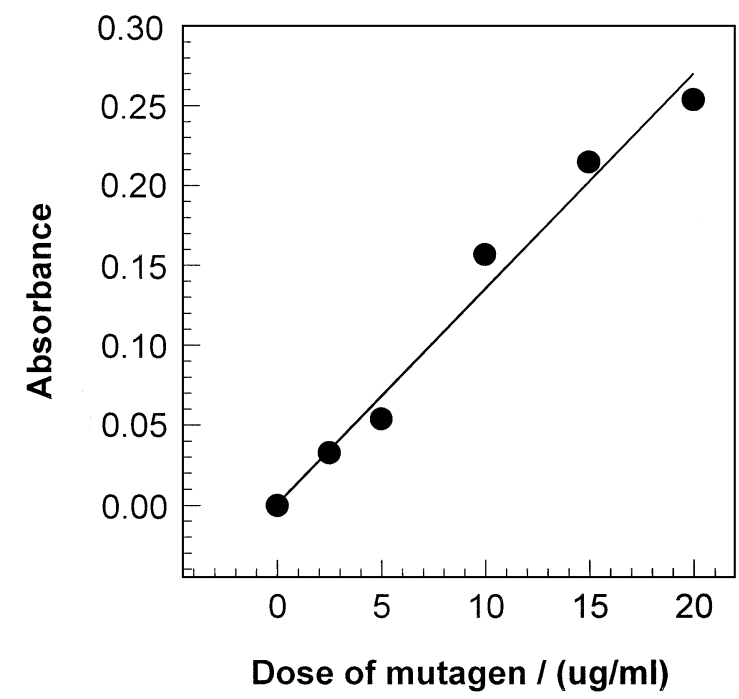

Fig. 7 Relationship curve between the absorbance and the dose of the mutagen.

Relationship between the absorbance and the dose of a mutagen After the absorbance values with the addition of different doses of DMS were obtained under selected experimental conditions, a calibration graph between the absorbance and the dose of the mutagen was acquired. The curve is shown in Fig. 7. It should be noted that a larger absorbance value could be detected when the dose of the mutagen was more. This result illustrates that the absorbance is linearly related to the dose of DMS over the range $2.5-20 \mu \mathrm{g} / \mathrm{ml}$. The regression equation is $A=1.27 \times 10^{-3}+0.0134 C$ and the correlation coefficient is $0.980(n=5)$, where $A$ is the absorbance (no dimension) and $C$ is the dose of the mutagen in $\mu \mathrm{g} / \mathrm{ml}$. Therefore, in a certain range of concentration, the mutagenicity is stronger when the concentration of the mutagen is greater. In this case, revertants produced by mutation are more, and the absorbance value of the culture system is larger due to their growth. This result agrees with a result obtained by the Ames test. ${ }^{17}$

The bacterial concentrations were also detected after finishing 
Table 2 Final bacterial concentrations in the presence of different doses of DMS

\begin{tabular}{cc}
\hline $\begin{array}{c}\text { Doses of DMS/ } \\
\mu \mathrm{g} \mathrm{ml}^{-1}\end{array}$ & $\begin{array}{c}\text { Final bacterial concentration/ } \\
\text { cells ml } \mathrm{l}^{-1}\end{array}$ \\
\hline 0 & $3.7 \times 10^{2}$ \\
2.5 & $3.1 \times 10^{4}$ \\
5.0 & $5.0 \times 10^{4}$ \\
10 & $1.5 \times 10^{5}$ \\
15 & $2.0 \times 10^{5}$ \\
20 & $2.4 \times 10^{5}$ \\
\hline
\end{tabular}

a mutagenicity test; the results are given in Table 2. It should be noted that in a certain dose range of the mutagen, the final bacterial concentration increases along with an increase in the dose of the mutagen.

\section{Conclusion}

In this work, combining with the bacterial growth situation, spectrophotometry was successfully applied to study a mutagenicity test. It was found that the mutagenic case displays a similar growth situation in the presence of histidine. Experiments also examined the effect of the dose of the mutagen on the absorbance. The results demonstrated that there is a linear relationship between the absorbance and the dose of the mutagen in a certain range. Compared with other reported mutagenicity test methods, ${ }^{8-13}$ this method can shorten the test time and avoid cumbersome operations; it can also detect mutagen directly. It is thus rapid and convenient. Therefore, a mutagenicity test with spectrophotometry will be a useful method in environmental monitoring.

\section{Acknowledgements}

This work was supported by the National Natural Science Foundation of China.

\section{References}

1. J. McCann, E. Choi, E. Yamasaki, and B. N. Ames, Proc Natl. Acad. Sci., 1975, 72, 5135.

2. S. S. Epstein, K. Fijii, and S. Asahina, Environ. Res., 1979 , 19, 163.

3. P. H. Michael, R. C. Glen, W. P. Bruce, L. C. Charles, L. L. Arthur, F. B. JR. William, G. T. William, and R. T. S. Bernd, Environ. Sci. Technol., 1998, 32, 3502.

4. A. S. Jarvis, V. A. McFarland, and M. E. Honeycutt, Ecotoxicol. Environ. Saf., 1998, 39, 131.

5. M. G. Zemank, S. J. Pollard, S. L. Kenefic, and S. E. Hrudey, J. Air \& Waste Manage. Assoc., 1997, 47, 1250.

6. S. Monarca, A. Zanardini, D. A. Dalmiglio, E. Falistocco, P. Manica, and G. Nardi, Water Res., 1998, 32, 2689.

7. P. Wilcox and S. Williamson, Environ. Health Perspect., 1986, 69, 141.

8. B. N. Ames, J. McCann, and E. Yamasaki, Mutat. Res., 1975, 31, 347.

9. D. M. Maron and B. N. Ames, Mutat. Res., 1983, 113, 173.

10. S. Lee, M. Suzuki, M. Kumagai, H. Ikeda, E. Tamiya, and I. Karube, Anal. Chem., 1992, 64, 1755.

11. J. Lewtas, S. Goto, K. Williams, J. C. Chuang, B. A. Peterseen, and N. K. Wilson, Atomos. Environ., 1987, 21 , 443.

12. I. Karube, T. Matsunaga, T. Nakahara, and S. Suzuki, Anal. Chem., 1981, 53, 1024.

13. I. Karube, T. Nakahara, T. Matsunaga, and S. Suzuki, Anal. Chem., 1982, 54, 1725.

14. X. Y. Zhang, "Shiyong Huanxue Shouce (Handbook of Practical Chemistry, in Chinese)", 1986, National Defence Industry Publishing House, Beijing, 1327.

15. J. L. Shi, "Huanjing Weishengwuxue (Environmental Microbiology, in Chinese)", 1993, East China Normal University Press, Shanghai, 385.

16. N. K. Zhu, in Proceedings of the Third Joint Conference of Air Pollution Studies in Asian Areas, 1987, Japan Society of Air Pollution, Tokyo, 326 - 336.

17. N. K. Zhu, X. Y. Du, X. J. Xia, F. D. Xu, and M. Y. Fan, "Huanjing Huaxue", 1994, 3, 250. 